\title{
MINIMUM WETTING RATE ON WETTED-WALL COLUMN
}

\section{CCORELATION OVER WIDE RANGE OF LIQUID VISCOSITY-}

\author{
TSUYOSHI MUNAKATA*, KAZUNORI WATANABE \\ AND KenJiRo MIYASHITA \\ Department of Chemical Engineering, Kyushu University, \\ Fukuoka, 812
}

\begin{abstract}
Minimum wetting rates were obtained for glass and stainless steel tubes by using glycerolwater mixtures of various compositions. Results show that the wetting behavior of the liquid in the range of high viscosity is sharply different from what has been reported previously by several workers.

To account for this phenomenon, the model proposed to date, in which only the inertia force is taken into account, is modified for addition of the viscous force. As a result, the following semiempirical equation is obtained:

$$
K\left(\frac{\Gamma}{\mu}\right)^{2 / 3}+\frac{2 \cdot 3^{5 / 3}}{15}\left(\frac{\Gamma}{\mu}\right)^{5 / 3}=A\left(\frac{\rho \sigma^{3}}{\mu^{4} g}\right)^{1 / 3}
$$

where $K$ and $A$ are the numerical constants to be evaluated appropriately.

It has been demonstrated that the proposed equation gives good correlation in a wide range of $\left(\rho \sigma^{3} / \mu^{4} g\right)$.
\end{abstract}

\section{Introduction}

In the previous work ${ }^{4)}$, in the absence of heat and mass transfer the minimum wetting rates (M. W. R.) were measured for smooth glass tubes by use of three liquid systems. Then it was concluded that the M. W. $R$. is strongly affected by the top shape of wettedwall, i. e. by the liquid distribution at the top, but that for the same tube with a given top shape a dimensionless equation of correlation has been obtained between M. W. R. and liquid physical properties as follows:

$$
\Gamma / \mu=C\left(\rho \sigma^{3} / \mu^{4} g\right)^{1 / 5}
$$

for $10^{7}<\left(\rho \sigma^{3} / \mu^{4} g\right)<10^{11}$.

The present work has been carried out to correlate over a wide range of $\left(\rho \sigma^{3} / \mu^{4} g\right)$, i. e. $10^{-2}<\left(\rho \sigma^{3} / \mu^{4} g\right)$ $<10^{11}$, by use of the glycerol-water system. Analytical treatment is also carried out with a modified model for wetting behavior of liquid, in which a viscous force affecting M. W. R. in the range of high liquid viscosity is taken into account. The resulting equation with two numerical constants shows good agreement with the experimental data in a wide range of $\left(\rho \sigma^{3} / \mu^{4} g\right)$ by means of an appropriate evaluation of the constants.

\section{Experimental Apparatus and Procedure}

The experimental apparatus and operating proce-

Received March 31, 1975. dure used in the present study are the same as those described in detail in the previous paper ${ }^{4)}$. For the test surface, six specimen tubes made of stainless steel were added to the six types of glass tubes used in the previous work. Each stainless steel tube has an outer diameter of $3.4 \mathrm{~cm}$ and length of $15.0 \mathrm{~cm}$, but each has a different shape of top similarly to that for glass tubes. These are shown in Fig. 1.

Measurements of M. W. R. were carried out at room temperature by using glycerol-water mixtures of various compositions in Case I, where the flow rate is reduced until the uniform film just starts to rupture, and in Case II, where the rate is increased until the initially dry surface is wetted completely.

\section{Experimental Results and General Observations}

The M. W. R. was measured mainly in Case II and

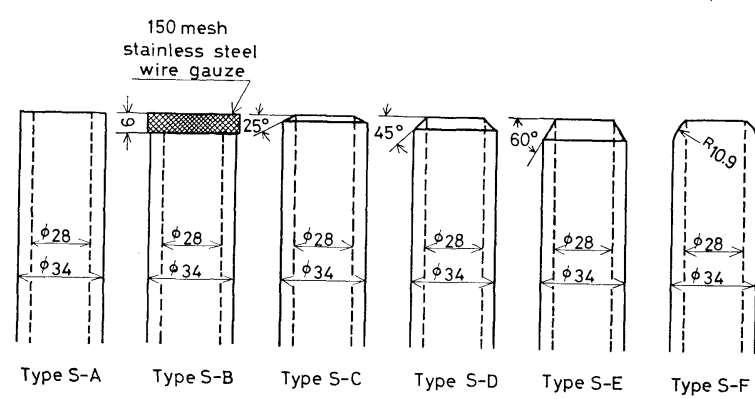

Fig. 1 Various types of top of stainless steel wetted-wall column 


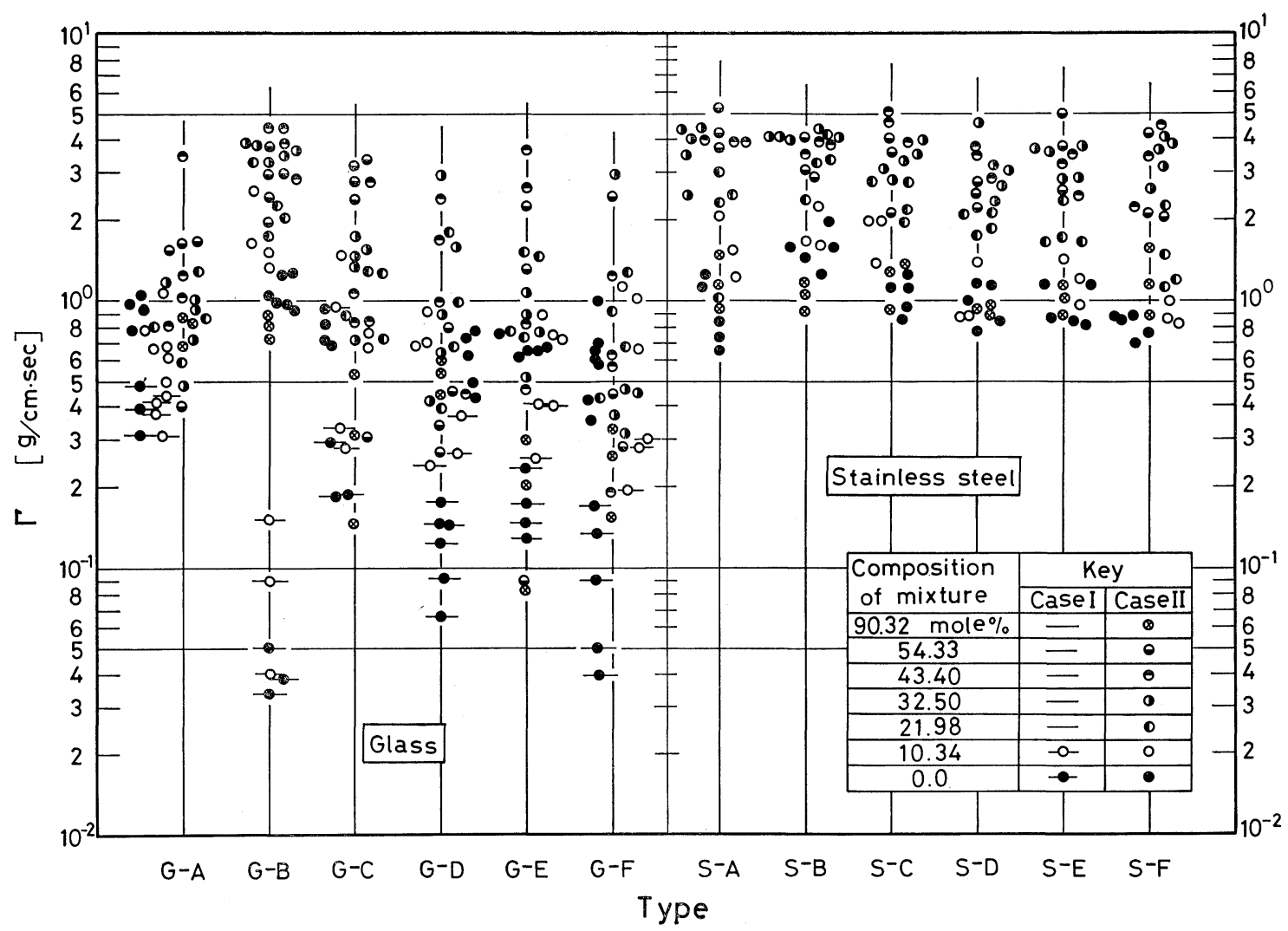

Fig. 2 Minimum wetting rates for each type of tube at room temperature

partly in Case I. Fig. 2 shows the typical data plotted as $\Gamma$ against the types of tube.

In Case I, the M. W. R. values for the glass tubes could be measured only at low concentration of glycerol ( 0 and $10.34 \mathrm{~mole} \%$ ), and were almost zero for the rest. This is similar to that found in the previous work. For the stainless steel tubes, the M. W. R. for all liquids were practically zero. This fact may be explained by the fact that stainless steel tube has a rougher surface than glass tube and liquid film formed on such a surface is likely to hold in the concavity.

In Case II, the values for stainless steel tubes are, as seen from Fig. 2, greater than those for glass tubes as a whole. It is noteworthy that the M. W. R. for stainless steel tubes are not only less affected by the wetted-wall top shapes but also show less scattering in value than for glass tubes. These points also probably result from the roughness of the stainless steel tubes, which causes a capillary action. The M. W. R. may be subject to this action rather than the end-effect of wetted-wall for this kind of tube, and the capillary action may not be very much influenced by a trace material adsorbed on its surface.

In the glycerol-water system used here, the liquid viscosity varies widely with glycerol concentration but its density and surface tension are little altered. So by taking M. W. R. and liquid viscosity to ordinate and abscissa respectively, data obtained for Type G-A and
Type S-A are shown in Fig. 3. According to Eq. (1), M. W. R. will be proportional to $\mu^{0.2}$. The present M. W. R. data in the low-viscosity range seem to satisfy this relation, but they take a maximum at about $1 \mathrm{~g} /$ $\mathrm{cm} \cdot \mathrm{sec}$ of viscosity and drop abruptly as the viscosity increases further. Data of Hobler et al. ${ }^{2)}$, which were obtained for stainless steel and glass flat plates by use of glycerol-water mixture, are also plotted on this figure. Their results also suggest that the M.W.R. does not always increase but decreases with increase of viscosity. It was also confirmed by a preliminary plot of $(\Gamma / \mu)$ against $\left(\rho \sigma^{3} / \mu^{4} g\right)$ that Eq. (1) is unsatisfactory in the high-viscosity range (such a plot is presented in the latter section in comparison with some quantitative equations, Fig. 5). Those described above denote that wetting behavior in the highviscosity range is different from that in the lowviscosity range. To illustrate this phenomenon, a physical model is set up in the following section.

\section{Physical Model}

A model is now developed for a laminar gravity flow of liquid film over a vertical flat plate to predict film breakdown. Consider an idealized case shown in Fig. 4, where a uniform stream with mean velocity $\bar{u}$ flows through the upper edge $\mathrm{AB}$ and when at point $\mathrm{S}$ a stable dry patch is formed downward the stream disrupts in two. This model is, essentially, the same 


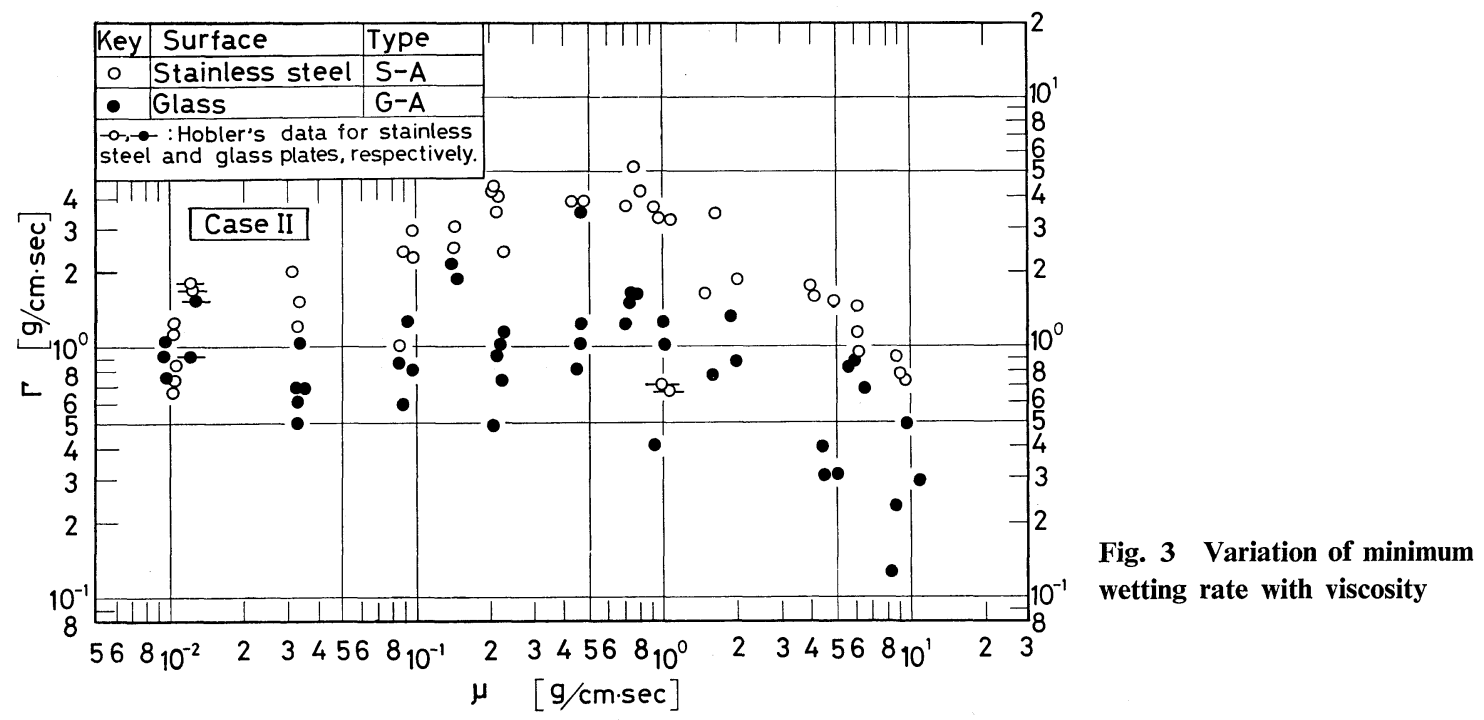

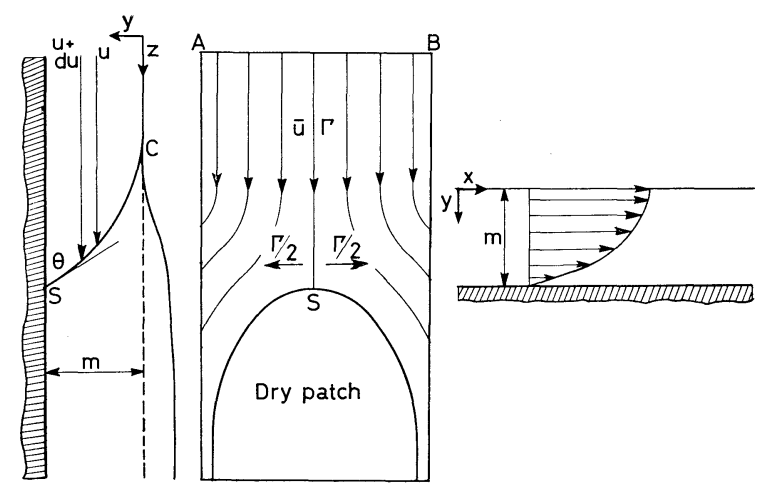

Fig. 4 Physical model and coordinate system

as that developed by Ponter et al. ${ }^{3}$. They have derived the following equation as a result of balancing the surface tension force along SC to the rate of change of momentum of the liquid in coming to rest. That is,

$$
\int_{0}^{m} \rho u^{2} d y=\sigma(1-\cos \theta)
$$

where $m$ is the film thickness and $u$ is the liquid velocity at a distance $y$ normal to the surface. In practical flow, however, the liquid flows transversely after bumping into a stagnation point of the dry patch. Consequently, a friction force of flow is caused in the horizontal direction, and its reaction force, $R$, tends to wet the dry patch. With assumption of a steady forced laminar flow in the transverse direction (see Fig. 4) one obtains $R=(3 / 2) \mu x \bar{u} / m$ and with further assumption* that $x$ is proportional to $m R$ is reduced to $K_{1} \mu \bar{u}$, where $K_{1}$ is a proportional constant and $x$ corresponds to a length in which the reaction force is extending.

\footnotetext{
* This assumption leads to $x \propto(3 \mu \bar{u} / \rho g)^{1 / 2}$ after Eqs. (5) and (6) are applied. And this proportionality seems fairly reasonable, because one can easily presume that the larger $\mu \bar{u}$ and the smaller $\rho g$ result in the greater $x$.
}

Therefore, $R$ is now added on the left side of Eq. (2) to modify Ponter's model as follows:

$$
K_{1} \mu \bar{u}+\int_{0}^{m} \rho u^{2} d y=\sigma(1-\cos \theta)
$$

In a uniform laminar film flowing over a vertical flat plate, the velocity $u$ at $y$, the mean velocity $\bar{u}$ and the film thickness $m$ are given by

and

$$
\begin{aligned}
& u=\frac{\rho g}{2 \mu}\left(m^{2}-y^{2}\right) \\
& \bar{u}=\Gamma / \rho m
\end{aligned}
$$

$$
m=\left(3 \Gamma \mu / \rho^{2} g\right)^{1 / 3}
$$

Substituting Eqs. (4), (5) and (6) into Eq. (3) and algebraic rearrangement give

$$
K\left(\frac{\Gamma}{\mu}\right)^{2 / 3}+\frac{2 \cdot 3^{5 / 3}}{15}\left(\frac{\Gamma}{\mu}\right)^{5 / 3}=A\left(\frac{\rho \sigma^{3}}{\mu^{4} g}\right)^{1 / 3}
$$

where $K=K_{1} / 3^{1 / 3}$ and $A=(1-\cos \theta)$.

On the left side of the above equation, the first term is owing to the viscous force and the second term to inertia force. Hence, for a considerably high-viscosity liquid the second term may be negligible, and conversely for a low-viscosity liquid the first term is negligible and then Eq. (7) is reduced to Ponter's equation.

\section{Discussion}

To test the validity of the model developed here, the typical M. W. R. data for glass tube (Type G-A) and stainless steel tube (Type S-A) in Case II are plotted in Fig. 5 as $(\Gamma / \mu)$ against $\left(\rho \sigma^{3} / \mu^{4} g\right)$. On the same figure are also plotted Hobler's data described in the previous section. The consistency between them and present results is satisfactory at $\left(\rho \sigma^{3} / \mu^{4} g\right)$ $=10^{10}$ but there is a little disagreement at small $\left(\rho \sigma^{3} / \mu^{4} g\right.$ ) (about $\left.3 \times 10^{2}\right)$.

The procedure adopted here for testing validity of Eq. (7) is to evaluate the value of $A$ as constant 
Fig. 5 Relation between $(\Gamma / \mu)$ and $\left(\rho \sigma^{3} / \mu^{4} g\right)$

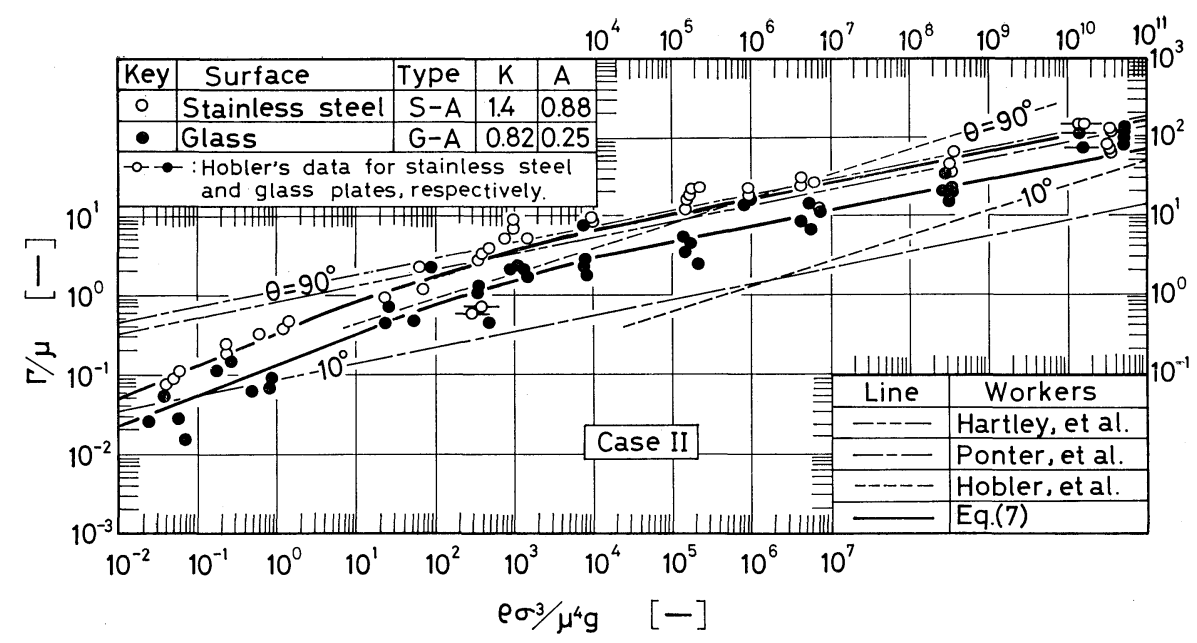

by Eq. (7), which shows that $\Gamma / \mu$ is proportional to $\left(\rho \sigma^{3} / \mu^{4} g\right)^{0.2}$ for the inertia force-controlling range and $\left(\rho \sigma^{3} / \mu^{4} g\right)^{0.5}$ for the viscous force-controlling range, and these lead to the conclusion that $\Gamma$ is proportional to $\mu^{0.2}$ and $\mu^{-1}$ respectively. And this is recognized in Fig. 3.

The larger power (0.325) in Hobler's equation may be because their experiments come up to the range where the viscous force plays a role. In Fig. 5 the equations reported by the other workers are also superposed to make it evident that for $10^{3}<\left(\rho \sigma^{3} / \mu^{4} g\right)$ $<10^{11}$ Ponter's and Hartley's equations show the same tendency as Eq. (7) and the former with $\theta=90^{\circ}$ agrees with the present results for stainless steel tube, while for $10^{1}<\left(\rho \sigma^{3} / \mu^{4} g\right)<10^{4}$ Hobler's with $\theta=90^{\circ}$ is consistent with the data for glass tube.

For the other types of wetted-wall column used in this work, the values of $A$ and $K$ were determined in the same manner described above and are given in the table in Fig. 6. These values of $A$ and $K$ thus obtained show that, as described above, the value of $A$ is dependent not only on the surface properties (accordingly the contact angle) but also on the wettedwall top shape. The values of $K$ are also conflicting, though there is no clear reason for its variation at the present. Fig. 6 shows the result plotted as $(\Gamma / \mu)_{\text {cal }}$ against $(\Gamma / \mu)_{\exp }$, which are calculated by Eq. (7) and experiment respectively. As seen from this figure, most of the results present good agreement between them as a whole.

\section{Conclusions}

Under the isothermal condition the M. W. R. for two kinds of test surface, i. e. glass and stainless steel, have been measured in Case I and Case II by using glycerol-water mixtures of various compositions. It is pointed out in Case II that the M. W. R. is lower than that expected by Eq. (1) in the range where the value of $\left(\rho \sigma^{3} / \mu^{4} g\right)$ is small. To illustrate this phenom- 


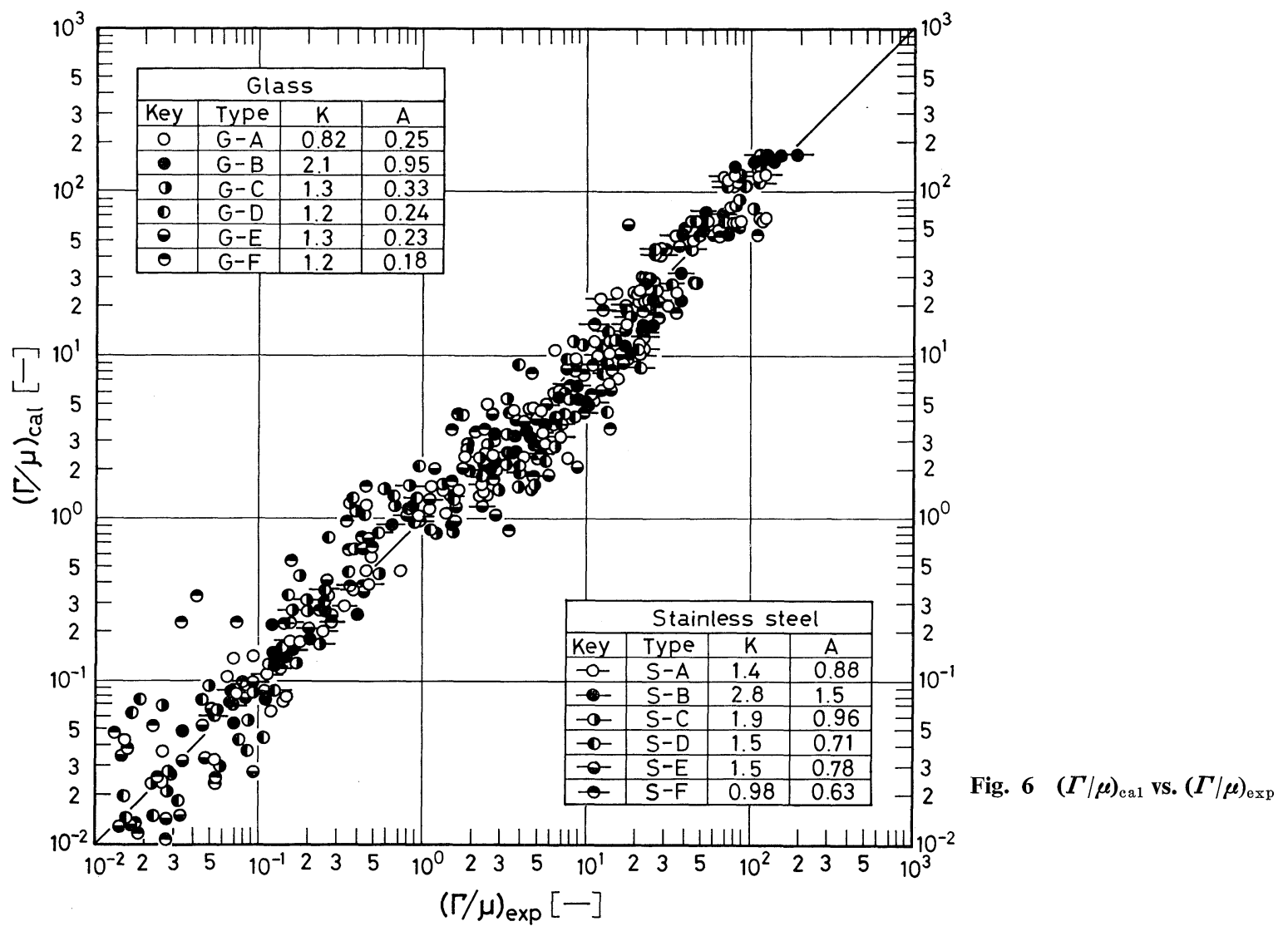

enon, the model proposed by Ponter et al. previously was modified by taking account of a viscous force in addition to the inertia force. Eq. (7) with two numerical constants was obtained on the basis of some reasonable assumptions.

It has been demonstrated that Eq. (7) gives a good correlation in the wide range of $\left(\rho \sigma^{3} / \mu^{4} g\right)$ by means of appropriate evaluation of the two constants.

\section{Nomenclature}

C

$g$

$K$

$K_{1}$

$m$

$R$

$u$ $=(1-\cos \theta)$

$=$ numerical constant defined in Eq. (1)

$=$ acceleration due to gravity

$=$ numerical constant defined in Eq. (3) [-]

$=$ Nusselt film thickness

$=$ reaction force

$=$ velocity of liquid at $y$ $\bar{u} \quad=$ mean velocity of liquid

$[\mathrm{cm} / \mathrm{sec}]$

$x=$ length where reaction force is extending [cm]

$y, z \quad=$ rectangular coordinates, see Fig. $4 \quad$ [cm]

$\Gamma \quad=$ minimum wetting rate $\quad[\mathrm{g} / \mathrm{cm} \cdot \mathrm{sec}]$

$\theta \quad=$ contact angle $\quad[-]$

$\mu \quad=$ viscosity of liquid

$\rho \quad=$ density of liquid

$\sigma \quad=$ surface tension of liquid

$[\mathrm{g} / \mathrm{cm} \cdot \mathrm{sec}]$

$\left[\mathrm{g} / \mathrm{cm}^{3}\right]$

[dyne $/ \mathrm{cm}$ ]

\section{Literature Cited}

1) Hartley, D. E. and W. Murgatroyd: J. Heat \& Mass Transfer, 7, 1003 (1964).

2) Hobler, T. and J. Czajka: Chemia Stosowana Ser. B, 2, 169 (1968).

3) Ponter, A. B., G. A. Davies, T. K. Ross and P. G. Thornley: J. Heat \& Mass Transfer, 10, 349 (1967).

4) Watanabe, K., T. Munakata and A. Matsuda: J. Chem. Eng. Japan, 8, 75 (1975). 